\title{
Aproximaciones \\ AL CENTRO HISTÓRICO DESDE LA CENTRALIDAD Y LA MULTIPLICIDAD
}

Approaches to the historic center from centrality and multiplicity

Rubén Romero-Carmona *, Sergio González-López **

Fecha de recibido:

11 Diciembre 2020

Fecha de aceptado:

11 Marzo 2021

*Universidad Autónoma del Estado de México, México. apouaemex@gmail.com

**Universidad Autónoma del Estado de México, México. gonzalezlop.sergio@gmail.com

RESUMEN. El propósito del artículo es abordar el análisis de los centros históricos desde su centralidad como su multiplicidad en el espacio urbano. Para tal fin, se integra en tres partes: la primera es la centralidad espacial, posteriormente sus atributos culturales-patrimoniales, y finaliza con el tratamiento de la multiplicidad de los centros históricos. El planteamiento central es que los centros históricos son espacios dinámicos en el territorio, relacionales entre sí y con la estructura urbana, y que constituyen espacios de comunidad que fusionan sentidos.

Palabras clave: centralidad, centro histórico, comunidad, espacio público, multiplicidad.

ABSTRACT.The purpose of the article is to address the analysis of historical centers from their centrality as their multiplicity in urban space. To this end, it is integrated into three parts: the first is spatial centrality, later its cultural-patrimonial attributes and ends with the treatment of the multiplicity of historical centers. The central thesis is that historical centres are dynamic spaces in the territory, relational to each other and to the urban structure and that constitute community spaces that fuse senses.

Key words: centrality, historic center, community, public space, multiplicity. 

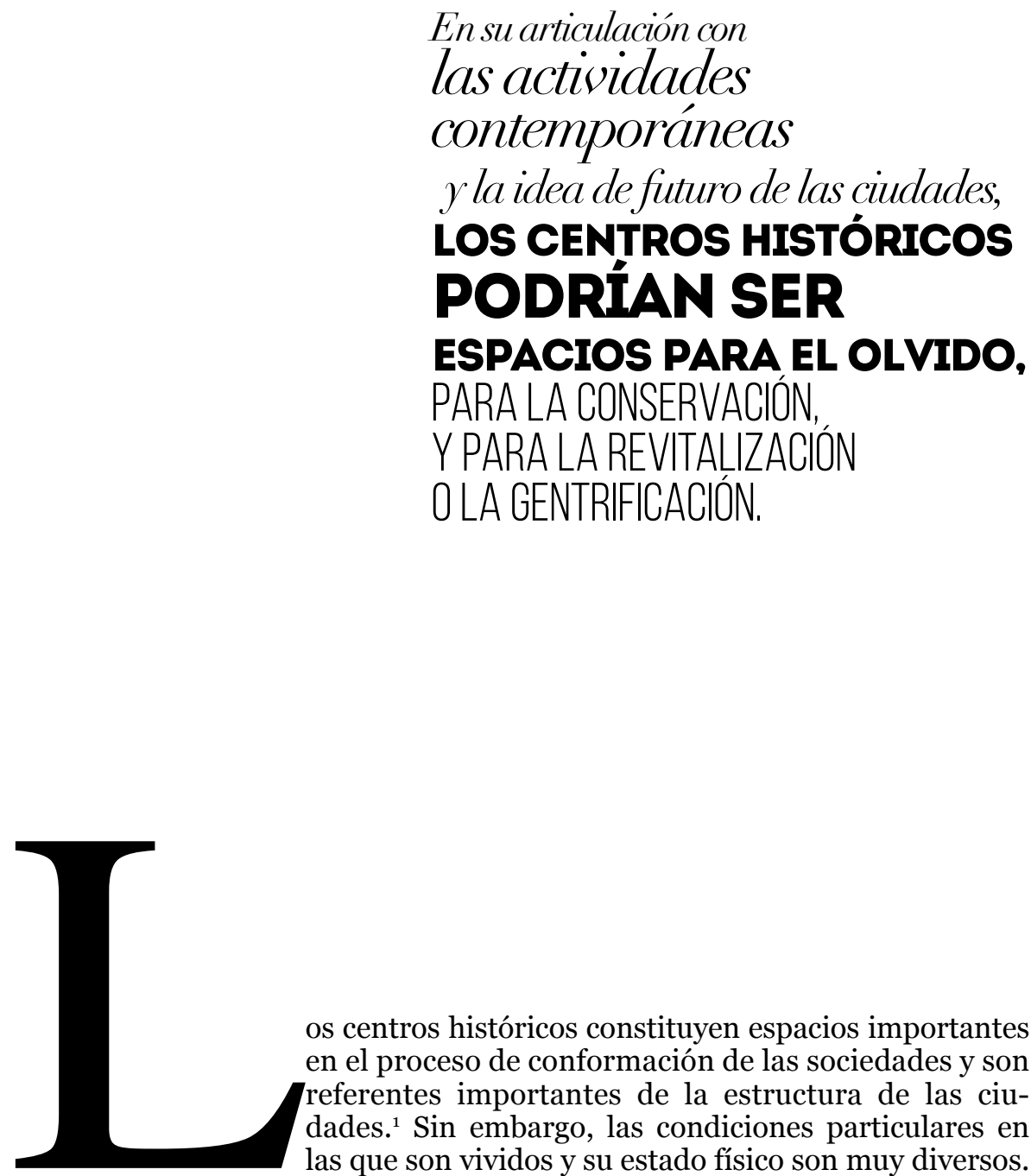

os centros históricos constituyen espacios importantes en el proceso de conformación de las sociedades y son referentes importantes de la estructura de las ciudades. ${ }^{1}$ Sin embargo, las condiciones particulares en las que son vividos y su estado físico son muy diversos. Desde espacios de identidad y orgullo que muestra las raíces históricas de la ciudad, o simplemente lugares para la realización de transacciones comerciales y de servicios, hasta sitios decadentes, con diversos avances de destrucción e inseguros, por los cuales es recomendable transitar con cuidado, y, por supuesto, las mezclas de estos y otros atributos que hace difícil poder denominarlo. Son espacios que han conseguido mantenerse en la centralidad de la vida urbana o bien han sido desplazados por modernos subcentros urbanos que atraen a las poblaciones de mayores ingresos, dejando para el centro histórico las actividades comerciales de mayoreo o para los sectores poblaciones de menor ingreso. En su articulación con las actividades contemporáneas y la idea de futuro de las ciudades, los centros históricos podrían ser espacios para el olvido, para la conservación, y para la revitalización o la gentrificación.

En general, la mayoría de los trabajos sobre los centros históricos privilegian el análisis de su patrimonio inmobiliario y la mezcla de funciones, y no tratan la cuestión de modelos espaciales que pudiesen arrojar algunas pistas para explicar su centralidad en la estructura urbana; asimismo, predominantemente otorgan la exclusividad de centro histórico a un solo espacio al interior de las principales ciudades, dejando de lado la cuestión de la multiplicidad de centros históricos en una gran ciudad, así como la constitución de estos centros en localidades más pequeñas.

\footnotetext{
1 Carrión (2001: 29) plantea que la relevancia del interés hacia los centros históricos radica en factores como el creciente deterioro que están sufriendo, la concientización para promover su desarrollo y remodelación, y una nueva tendencia de urbanización de las ciudades latinoamericanas hacia el "regreso a la ciudad construida".
} 
El presente artículo tiene como objetivo tratar los centros históricos a través del abordaje de su centralidad como su multiplicidad en el espacio urbano. Con base en esto se explora el planteamiento de la posibilidad de ver a los centros históricos como dinámicos en el territorio, relacionales entre sí y con la estructura urbana y que constituyen espacios de comunidad que fusionan sentidos. Por tal motivo, el trabajo se conforma por tres partes: las dos primeras referidas a la centralidad espacial y las cuestiones culturales-patrimoniales de los centros históricos; y la tercera a la multiplicidad de los centros históricos.

\section{LA CENTRALIDAD ESPACIAL Y DE LOS CENTROS HISTÓRICOS}

El término centro, que proviene del latín centrum y del griego kévtpov, se refieren a la relación que existe entre el origen del círculo y el contexto o perímetro, y que por analogía hace referencia al punto inicial y central del espacio urbano (Krafta, 2008: 15). Por tanto, se puede interpretar como un punto relacional, es decir, adquiere sentido solo cuando se le relaciona con otros puntos o áreas.

En torno al espacio se han desarrollado diversos modelos que pretenden explicar la localización teniendo como eje el centro y la centralidad. La teoría de localización, propuesta por Johann Heinrich Von Thünen, en 1820, es el principio teórico de la centralidad, puesto que considera a la centralidad como un punto central y de gran importancia dentro del territorio; además, el espacio central actúa como concentrador económico, a través del mercado se realizan las mayores transacciones de productos (Krafta, 2008: 3). En el modelo de Von Thünen solo existe una centralidad absoluta, que funciona como el origen de la estructura urbana económica, de aquí la importancia de la funcionalidad del centro histórico, este espacio se consolida como el único centro de la ciudad; también el espacio central actúa como un mercado de atracción por su jerarquía, consumo e influencia territorial.

La teoría contextualiza, un periodo donde existía una distribución territorial a base de producción agrícola y actividades económicas que giraban alrededor del "mercado" (Camagni, 2005: 52). Todas estas características ejemplifican a la primera fase del ciclo de vida urbano, con un patrón monocéntrico de la historicidad.

La distribución concéntrica del modelo de Von Thünen, aunque no es nada reciente, destaca la importancia de la localización respecto de la centralidad, a mayor distancia del espacio central se tiene un menor valor económico, los precios del suelo disminuyen del centro a la periferia (Duch, 2008: 6), esto se expresa a través de las curvas de renta, además los usos y costos del suelo están en función de la localización y de las capacidades económicas. Si esto lo enfocamos a un centro histórico, el contexto es significativo en el sentido del desaprovechamiento del suelo, ocasionado por el aumento de costos del suelo, pero en la disminución de inversión para su aprovechamiento.

Ya en 1993, Walter Christaller formula la teoría del lugar central, en este modelo destaca el valor de las centralidades y de la concentración económica, por el funcionamiento económico de las ciudades (Krafta, 2008: 23); asimismo, el espacio central histórico está asociado a las actividades económicas, que atraen directamente a los asentamientos humanos; asimismo, el grado de centralidad se establece con el número de servicios y bienes que se ofrecen.

El centro histórico es el centro y distribuidor de toda la red con estructura de hexagonal o panal, es un modelo de equilibrio espacial; a partir de la centralidad surgen y se conectan con otras concentraciones económicas, las conexiones se crean a través de una red de áreas circulares, que se crean en torno a los centros de servicios o lugares centrales (Camagni, 2005: 98-109).

Los principios teóricos del modelo se rigen por tres factores clave: la jerarquía de los centros, la dimensión y cercanía de los centros, y el área de mercado de cada centro. La teoría propuesta por Christaller resalta la distribución espacial de los centros urbanos, con una jerarquía ordenada y coherente de los centros en conjunto, a través de las economías de escala; además, los asentamientos humanos actúan como centros óptimos de distribución para los servicios y mercancías en el sistema urbano espacial (Camagni, 2005: 98-109). Entonces, esta teoría se sustenta en la jerarquía de centros urbanos y están en función de las economías y deseconomías del espacio urbano, además de los usos y ocupación del suelo.

Desde la perspectiva del centro como un punto relacional con otros puntos o áreas, el centro actúa como un espacio concentrador, que tiene la capacidad de atraer e influir en la organización del ámbito espacial, su importancia varía según sus características y jerarquía, respecto a las condiciones del entorno y la relación que establece con otros espacios. Derivado de esta interpretación, el centro histórico se define como el punto medio y central del espacio urbano influyendo, a partir de las relaciones de vinculación y a la lógica de conectividad en la organización funcional de la ciudad (Soja, 2008: 9), representando, en el 
contexto de la ciudad actual, el núcleo original de la ciudad histórica.

La centralidad, entendida como la cualidad del centro, se establece como un punto primordial en el espacio, definido por la concentración económica, es decir, como un lugar de intercambio, producción y consumo de la ciudad (Weber citado en Lezama, 2010: 128) es, en este sentido, una concentración de materia urbana, un elemento esencial del sistema urbano que se ubican en lugares específicos.

De Mattos (1999) señala que, dentro de los cauces de la vertiente keynesiana, se abrieron paso las teorías del desarrollo económico y social, las que sin duda tomaron sus hipótesis fundamentales de los modelos de crecimiento, si bien los límites entre unos y otras no pueden ser establecidos con precisión. En este sentido, los modelos de crecimiento endógeno tienen como rasgo distintivo básico su estructuración en torno a una función de producción, donde la tasa de crecimiento depende básicamente del stock de tres factores: capital físico, capital humano y conocimientos o progreso técnico, que pueden ser objeto de acumulación y, además, generan externalidades. También destaca que los modelos de crecimiento endógeno establecen externalidades positivas que están relacionadas a la inversión, traducidos en retornos crecientes a escala, lo que equivale a afirmar que las que se derivan del aumento del stock de capital, incluyendo el capital humano. En síntesis, el nivel de ingreso a largo plazo en un centro histórico estaría determinado por la acumulación de capital físico, capital humano y conocimientos, donde los niveles respectivos pueden considerarse como variables endógenas, determinados por decisiones de ahorro e inversión motivadas por expectativas de ganancia.

Entendida así, siguiendo con De Mattos, la centralidad está integrada por tres componentes espaciales: los factores endógenos, que son todos los aspectos espaciales que interactúan dentro del área urbana, estos enfatizan el funcionamiento interno del centro histórico, en este lugar se potencializa el valor de las tierras por sus actividades; asimismo fuera del núcleo urbano existe una constante interacción entre los núcleos de transporte, empleo y comercio de toda la ciudad; los factores internos, derivados del núcleo urbano relacionado con los servicios y comercio; y los factores exógenos, que son determinantes en el funcionamiento complejo de la ciudad.

En la figura 1 se representa la centralidad endógena, como el punto central que origina la ciudad, la cual está cercanamente asociada con los suburbios, el área está delimitada por los límites de la ciudad. La figura de la centralidad exógena representa el núcleo urbano como área principal y la actividad comercial externalizada y sus interconexiones regionales.

De acuerdo con lo anterior, el centro histórico es representado como una concentración urbana, con asentamientos caracterizados por ser de formación histórica o por ser una reciente aglomeración de actividad económica. De acuerdo con Pineda (2010), actúa como polo de atracción para la población, por la

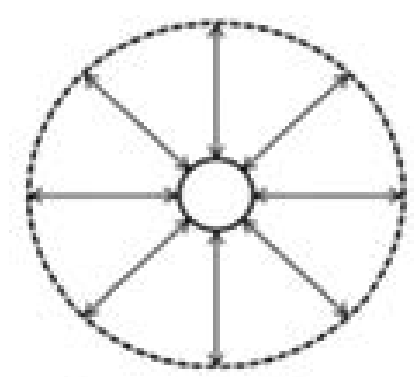

Centralidad endógena

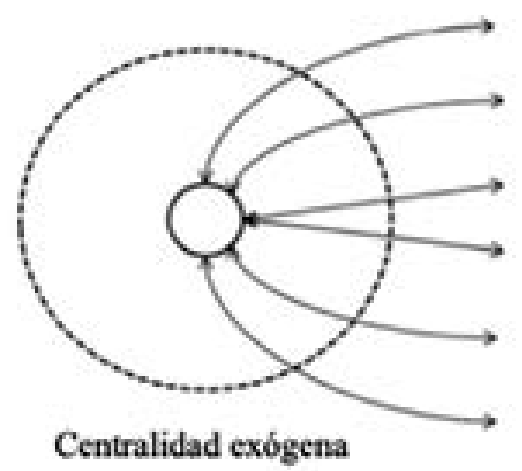

FIGURA 1. FACTORES DE LA CENTRALIDAD URBANA. FUENTE: ELABORACIÓN PROPIA CON BASE EN DE MATTOS (1999).

numerosa presencia de actividades vinculadas a los sectores de comercio, servicios, gobierno, administración pública; este espacio posee determinadas funciones urbanas centrales, que son necesarias para equilibrar el funcionamiento de la ciudad.

Considerando a la economía en el espacio regional se podría conceptualizar que una región económica es un área geográfica identificable caracterizada por una estructura particular de sus actividades económicas, con referencia a un conjunto de condiciones asociadas físicas y/o biológicas y/o sociales que presentan un alto grado de homogeneidad y que mantienen un cierto tipo de relaciones internas y con el exterior (Bassols, 1979: 306).

Los procesos de crecimiento y desarrollo implican multicausalidad que son resultado de interacciones entre subsistemas funcionales, y que a su vez dan origen a la formación de subsistemas: regiones o localidades. Con relación a ello, bajo la línea económica una región es una categoría que se refiere a un área continua, una unidad espacial con cierta 


\section{El concepto de desarrollo}

que se analiza puede concebirse

COMO UN PRODUCTO

DE acciones específicas

REALIZADAS EN

espacios regionales
POR ACTORES
CONCRETOS,
PERO DESTACANDO SU,
CARACTERISTICA HISTORICA.

homogeneidad interna, en la cual las relaciones que se establecen le dan cierta cohesión (Pineda, 2010: 204).

Para la llamada Nueva Geografía Económica (NGE), propuesta por Paul Krugman en 1991, ocupa hoy un lugar relevante en el ámbito de los análisis territoriales. Irrumpió con fuerza a principios de los noventa y se ha ido consolidando como un enfoque robusto e innovador en el estudio de las relaciones entre el espacio y los procesos económicos. La NGE ha servido para poner fin al largo silencio con que la corriente dominante del análisis económico había tratado cuestiones como dónde tiene lugar la actividad económica y cómo evoluciona; por qué tiende a concentrarse; y cuáles son las causas explicativas (Krugman, 2000).

La NGE, si bien, es una propuesta relativamente reciente, que se basa en teorías económicas anteriores, en realidad es un modelo que, al igual que los centros históricos, se ha ido conformando con relación al espacio-tiempo en que se desarrolla una ciudad. Las aportaciones recientes de la geográfica económica al centrarse en el papel de la aglomeración han puesto de relieve las interacciones entre costes de producción, tamaño de los mercados y decisiones de localización empresarial.

La cuestión a destacar en la NGE en la función del centro histórico es tratar de proporcionar alguna explicación a la formación de una gran diversidad de formas de aglomeración (o de concentración) económica en espacios geográficos. De acuerdo con Krugman (2000), la aglomeración o agrupación (clúster) de la actividad económica tiene lugar a distintos niveles geográficos y tiene una variedad de formas distintas (CuadradoRoura, 2013: 5-28). De acuerdo con esto, un ejemplo, podría ser el tipo de aglomeración que surge con la agrupación de pequeñas tiendas y restaurantes en un centro histórico.

En el otro extremo del espectro se encuentra la estructura centro-periferia de la economía global, correspondiente al dualismo norte-sur. Es asimismo importante señalar que todos estos tipos distintos de aglomeración, a diversos niveles, están a su vez insertos en una economía mayor, formando en su conjunto un complejo sistema (Cuadrado-Roura, 2013: 5-28).

Lo anterior, hace pertinente señalar, aunque sea brevemente la relación de estos procesos con uno más general, que sería la idea de "desarrollo" en su expresión de tiempo y espacio. El concepto de desarrollo que se analiza puede concebirse como un producto de acciones específicas realizadas en espacios regionales por actores concretos, pero destacando su característica histórica.

El concepto de desarrollo proviene de la raíz des, que a su vez proviene del latín dis, que quiere decir "aparte, separado, hacer lo contrario", o sea, una negación, en este caso antepuesta a la palabra arrollar, la cual significa "envolver en rollo", así; desarrollo quiere decir desenvolver o desplegar una potencialidad o una cualidad que ya existe en sí mismo de manera plegada o no expresada; desarrollar implica, por lo tanto, la existencia de un sujeto, el cual despliega una acción (Pineda, 2010: 199).

Es claro que la preexistencia de un modelo de desarrollo probado, funcional y con pretensiones de validez universal hace que cualquier otra alternativa se ubique en una posición de conflicto y aparezca como un intento de cambio, entendido éste como una amenaza con romper la funcionalidad probada y la estabilidad de las sociedades desarrolladas (Cuadrado-Roura, 2013: 5-28), debido a que su sistema de acción histórica y sus recursos económicos y sociales, además de los culturales, constituyen los elementos modelo para lograr el desarrollo de cualquier espacio que siga el camino trazado.

Esta realidad da pauta a ciertas cuestiones, pues no se ha solucionado el problema que aparece de manera polarizada, quedando en última instancia, caracterizado por posturas de tipo ideológico, las cuales han sido construidas mediante formas de acción histórica diferencial, aunque con pretensiones y perspectivas de funcionalidad para cada realidad histórica, es decir, con perspectivas de universalidad.

En lo general, la virtud de los diversos planteamientos anteriores, es que permite tener una visión espacial de los procesos territoriales, distinguiendo diversos atributos, 
entre ellos, una idea de centralidad con relación a otros puntos y superficies, que de alguna manera posibilitarían tener una perspectiva espacial relacional de los centros históricos. Pero, también muestran fuertes limitaciones, entre las que podría destacarse que las relaciones establecidas obedecen sobre todo a una racionalidad económica, donde todo es posible cuantificar y, por tanto, son insuficientes para poder comprender complejos procesos políticos, culturales y sociales que históricamente han constituido y transformado lo que entenderíamos como centros históricos.

\section{LA CUESTIÓN PATRIMONIAL-CULTURAL DE LOS CENTROS HISTORICOS}

El concepto de centro histórico es relativamente reciente; surge a partir de la propia crisis generada en estos sectores de la ciudad, cuando se vieron amenazados tras la reconstrucción de posguerra en Europa o por políticas urbanas desarrollistas y aculturadas, aplicadas allá y también en América Latina, más tardíamente (Rodríguez, 2008: 51). La acción de salvaguarda patrimonial ha transitado de una visión del monumento aislado a una comprensión del valor del conjunto urbano; en las primeras décadas con una visión de mecenazgo y a partir de los años 1990, entendiendo la dimensión económica de la recuperación de los centros históricos.

El centro histórico constituye una pieza fundamental en el paisaje urbano de la ciudad y su significado desborda ampliamente el papel que le correspondería en función de su superficie, entidad demográfica o actividad económica. Aunque es una parte pequeña en el actual tejido urbano, constituye el espacio histórico por excelencia y, en gran medida, la memoria colectiva de la ciudad. Camallonga (2012) menciona que es un producto histórico-social de carácter singular que contribuye a los rasgos excepcionales de un determinado paisaje urbano y que la ciudad tenga sus propias señas de identidad.

La importancia de los centros históricos radica en su capacidad de generar fuentes de empleo, impactar positivamente al turismo, la cultura y múltiples beneficios sociales, el desarrollo territorial se aprecia como una alternativa para alcanzar un desarrollo integralidad de estos espacios.

Desde el punto de vista funcional, los centros históricos se definen por un marcado carácter multifuncional, ya que en ellos conviven funciones residenciales, comerciales, religiosas, administrativos, lúdicas, etc. La diversificación de actividades aporta una gran riqueza a la vida urbana que en ellos se desarrolla. No obstante, esta riqueza se pierde, en buena medida, cuando el sector terciario penetra de forma desmedida o cuando quedan convertidos en espacios museos carentes de vida urbana.

Conceptualmente, el centro histórico tiene un doble significado relacionado a lo espacial y a lo temporal. Tiene carácter de centralidad con respecto a la ciudad, no siempre desde el punto de vista físico, pero sí desde la óptica funcional, además de haber sido escenario de hechos históricos relevantes acumulados a lo largo del tiempo.

Durante siglos, el centro histórico albergó prácticamente todas las funciones que caracterizan a una ciudad, en una racional mixtura de usos resueltos a través de tipologías arquitectónicas y urbanas específicas, expresadas bajo patrones estilísticos diferentes, que respondieran a la diversidad y dimensión de las necesidades citadinas (Rodríguez, 2008: 53).

Históricamente este hecho (el reconocimiento del centro histórico en su especificidad en el marco urbano de la ciudad) se produce con la aceleración del proceso de urbanización, en un contexto de modernización de la sociedad, impulsado por el proceso de industrialización. Este proceso de cambio acelerado genera una reacción de las élites locales, que se preocupan por lo que se pierde, reivindicando la creación de un marco institucional de defensa de este imaginario cultural nacional, donde "lo paradójico de la situación radica en que el nacimiento de la centralidad histórica se produce en el momento en que entra en decadencia" (Carrión, 2006: 174-175).

Desde la perspectiva monumental y patrimonial de los centros históricos formulada por asociaciones e instituciones, uno de los antecedentes relevantes es La Carta de Atenas de 1933, que plantea que el uso de los monumentos debe garantizar la continuidad de su existencia, planteándose acciones de restauración que no perjudiquen los estilos de ninguna época. Se acepta el empleo de nuevos materiales, pero se recomienda que sean disimulados, a manera de no alterar el carácter del edificio. Algo se enuncia sobre el respeto que los nuevos edificios deberán tener hacia la fisonomía de la ciudad conservada y sobre todo en la cercanía de los monumentos y a la necesidad de preservar ciertas perspectivas particularmente pintorescas (CIAM, 1933).

Pero se puede afirmar que la preocupación por la preservación de los inmuebles históricos se expresa por primera vez en un documento de impacto internacional cuando se redacta la Carta de Venecia de 1964, donde se planteó la noción de patrimonio histórico asociada al sitio urbano o rural que da tes- 
timonio de una civilización particular, de una evolución significativa o de un acontecimiento histórico, comprendiendo no sólo las grandes creaciones, sino también las obras modestas que han adquirido con el tiempo significación cultural (ATM, 1964).

Para América Latina y el Caribe, sobre todo para los países andinos, la denominada Las normas de Quito, mejor conocida como Carta de Quito de 1967, tiene una gran relevancia en el reconocimiento de la importancia de los centros urbanos, al resaltar la problemática que les afecta como la necesidad de acciones para enfrentarla y revalorizarlos (UNESCOPNUD, 1977: 1-10). Sus planteamientos fueron reforzados en el Coloquio sobre la preservación de los Centros Históricos ante el crecimiento de las ciudades contemporáneas, también realizado en Quito en 1977, y, en sus conclusiones define a los centros históricos como "todos aquellos asentamientos humanos vivos, fuertemente condicionados por una estructura física proveniente del pasado, reconocibles como representativos de la evolución de un pueblo. Como tales se comprenden, tanto los asentamientos que se mantienen íntegros desde aldeas a ciudades, como aquellos que a causa de su crecimiento, constituyen hoy parte de una estructura mayor"; que "por sí mismos y por el acervo monumental que contienen, representan no solamente un incuestionable valor cultural sino también económico y social”; a la vez que "no sólo son patrimonio cultural de la humanidad sino que pertenecen en forma particular a todos aquellos sectores sociales que los habitan" (UNESCO-PNUD, 1977: 11).

La ciudad de Quito, en 2016, fue sede de dos importantes eventos en materia urbana: La Conferencia de las Naciones Unidas sobre la Vivienda y el Desarrollo Urbano Sostenible (Hábitat III) (onU, 2016) y el Foro Alternativo a Hábitat III (Borja, Carrión y Corti, 2016), convocado por multitud de personajes y organizaciones de la sociedad civil. En ambos eventos no se hizo referencia a los centros históricos. En la Conferencia, el eje fue los Objetivos del Desarrollo Sustentable aprobado el año previo por la Organización de las Naciones Unidas (ONU); mientras que, en el Foro, la tónica fue la crítica sustantiva a la Conferencia y al posicionamiento y forma de trabajo de la propia onu en materia urbana, como la formulación de planteamientos relevantes alternativos en lo urbano $\mathrm{y}$ territorial para América Latina.

Vale la pena poner la alerta si este relativo desinterés hacia los centros históricos estará marcando una tendencia de largo a la baja, por lo menos de las instituciones internacionales. Por otra parte, es notoria la multiplicidad de trabajos relevantes que tratan acerca de los centros históricos de América Latina y el Caribe, entre ellos, sobresalen los textos de Fernando Carrión (2001, 2005 y 2019),
Balandrano, Valero y Ziccardi (2016), René Coulomb (2001, 2019) y Gonzalo Soltero (2009).

\section{LA MULTIPLICIDAD DE LOS CENTROS HISTÓRICOS}

Las ciudades latinoamericanas, históricamente han sido una noción centralista en prácticamente todos los órdenes y su capacidad ha predominado de manera marcada durante los siglos. Para el caso de las ciudades de México concentran significativamente las actividades económicas y la población a la vez que desborda su crecimiento físico hacia zonas conurbadas y, regionalmente adquieren una influencia directa sobre la economía de las ciudades del centro del país.

El proceso urbano en México se soporta de manera desigual sobre su territorio, siendo distinguibles espacios que concentran actividades económicas orientables hacia el mercado externo, de otros que destinan su producción de manera predominante hacia el mercado interno; estos espacios son ámbitos de tensión social, económica y ambiental y presentan sobre todo una expresión radial o de ejes, convergiendo del centro de la ciudad. La economía se concentra sobre todo en el espacio central, el cual acumula alrededor de $1 / 3$ del empleo y cerca del $40 \%$ del valor agregado de la megalópolis (González, 2004:59-64).

Ante la crisis del modelo económico como el tránsito hacia la apertura económica y el neoliberalismo, la función económica de las ciudades de México tiende hacia un replanteamiento sustancial: una reestructuración económica como la ampliación territorial de su influencia (González, 2004:75-84). Esto, para el caso de la Ciudad de México, supone que su área urbana ha rebasado el límite político-administrativo del entonces Distrito Federal-Ciudad de México, desde febrero de $2017,{ }^{2}$ desde la década de los sesenta del siglo pasado hasta ser una megalópolis que se desarrolla sobre otras tres (Estado de México, Morelos, Hidalgo) y alcanza más de 21 millones de habitantes. Por lo que, no es posible suponer que el único centro histórico de esta Megalópolis son sólo dos perímetros ${ }^{3}$ localizados alrededor del llamado Zócalo.

2 Gobierno de la Ciudad de México (2017), Constitución Política de la Ciudad de México, Gaceta Oficial de la Ciudad de México, 5 de febrero de 2017 (Última reforma: 27 de noviembre de 2019).

3 Perímetro "A" patrimonial y un Perímetro "B" concebido como zona de protección del corazón patrimonial (Coulomb, 2019: 115). 


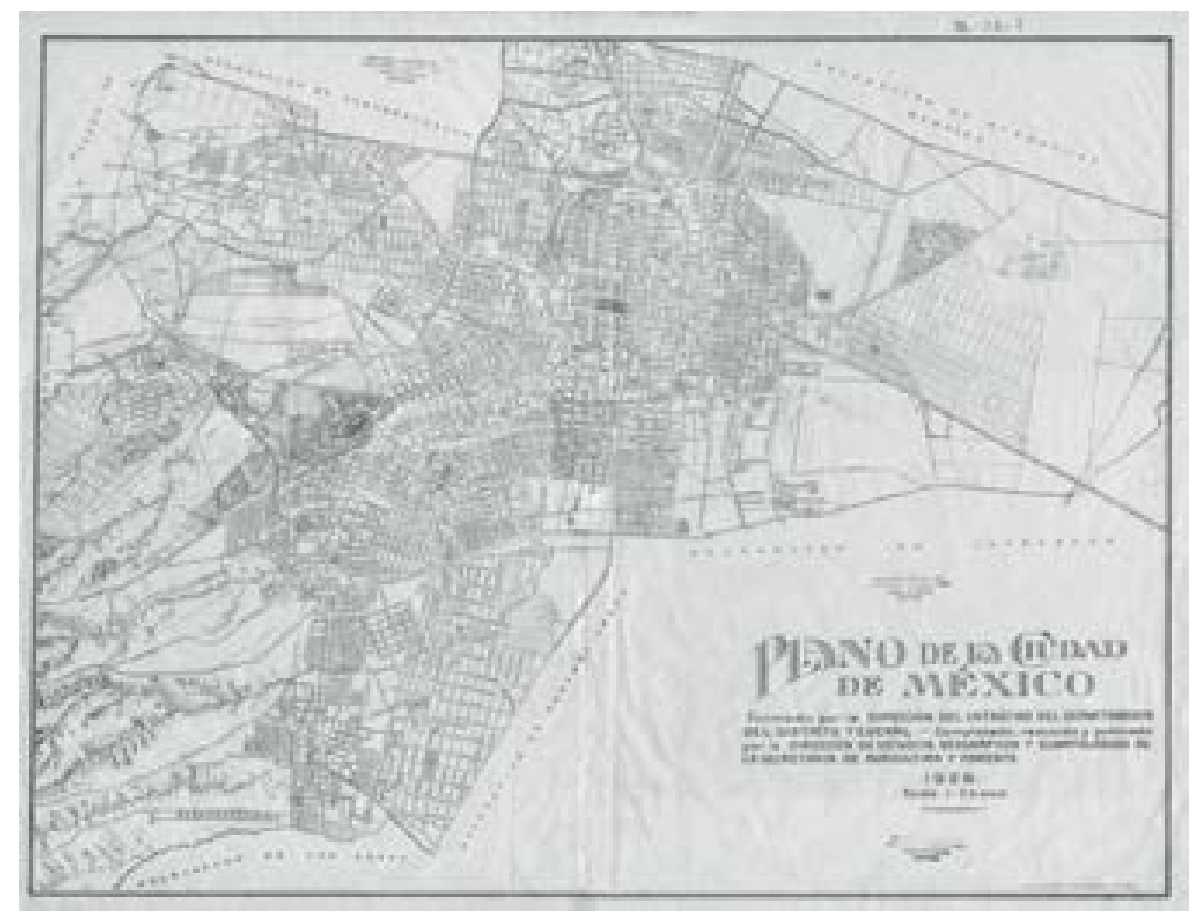

FIGURA 2. PLANO DE TRAZA URBANA CIUDAD DE MÉXICO, 1929.

FUENTE: MEDIATECA INAH, 1929.

Para el abordaje de este centro histórico para ejemplificar el planteamiento de la multiplicidad de centros históricos, es necesario tener presente algunos de los principales rasgos históricos de este ámbito territorial que es el altiplano mexicano. Si bien es aceptado que el origen de la Ciudad de México, data de 1325 con el establecido de los mexicas en una de las islas del sistema de lagos; fue el eje de un imperio que articuló a otros en la llamada Triple Alianza y extendió sus dominios en alrededor de $300000 \mathrm{~km}^{2}$, la más grande metrópoli que conoció Mesoamérica. También debe considerarse que, alrededor de esta zona existieron otros poblados importantes como los teotihuacanos, cuyos vestigios están inmersos en la Megalópolis de México.

En el plano de la Ciudad de México (figura 2) se observa la ampliación de los límites de la ciudad en las cuatro orientaciones, quedando el zócalo en el centro. Una porción de la zona central de la Alcaldía Cuauhtémoc, Ciudad de México, es donde se ubican edificios emblemáticos históricamente como el Palacio Nacional, la Catedral Metropolitana, la Plaza de la Constitución, el Templo Mayor (de origen mexica) y otros cientos de inmuebles y monumentos históricos patrimoniales sobre una superficie de alrededor de $9.7 \mathrm{~km}^{2}$ (Coulomb, 2001: 139), y es denominada oficialmente como el "Centro Histórico de la Ciudad de México" y reconocida como tal por habitantes y visitantes de esta ciudad. Reconociendo su innegable valor histórico, cultural, social y económico, no obstante, ¿̇podría suponerse que es el único sitio con valor histórico dicha megalópolis? Podríamos decir que no, y esta afirmación tiene diversas implicaciones de variados órdenes, que no podremos desarrollar en este trabajo, porque delinearemos solo una parte de ellos derivados de la posibilidad de reconocer múltiples centros históricos en la megalópolis.

Si tenemos como referente los planteamientos señalados en el Coloquio sobre la preservación de los Centros Históricos ante el crecimiento de las ciudades contemporáneas (UNESCO-PNUD, 1977: 11), se podría decir que otros espacios de la urbe cubren uno o algunos de los atributos necesarios, aunque no sea con las mismas dimensiones del centro histórico ya reconocido, como los casos de Coyoacán, San Ángel, Tlalpan, Xochimilco, Tlaltelolco o Teotihuacan, Cuicuilco, Otumba, entre otros. La cuestión no es si deban ser o no reconocidos oficialmente como centros históricos, sino más bien, qué representaría esta multiplicidad de centros históricos para el conjunto de la ciudad, su estructura urbana y habitantes.

Para el conjunto de la ciudad, daría una visión más amplia e integrada sobre su proceso histórico, que en ocasiones se ve reducida cuando la referencia está subordinada a solo el centro histórico reconocido oficialmente. Por supuesto, además podría incorporar otras posibilidades de vinculación entre los 
centros que han sido "absorbidos" por el crecimiento del área urbana. Haciendo distinguibles el valor y la funcionalidad de subcentros urbanos "históricos" de los "modernos" como Satélite, Polanco y Santa Fe. A la vez que, para los residentes podría reforzar una percepción de identidad que pudiese contribuir hacia la construcción de una ciudadanía más consciente de su entorno inmediato y en general, fusionando sentidos a la vida urbana que se da frente a frente.

En otros términos, el tratamiento de múltiples centros urbanos daría otra comprensión sobre procesos expresables en intersecciones de planos. En el sentido que, en los procesos históricos se van sobreponiendo planos de espacios que corresponden a diferentes épocas, estilos e incluso formas de organización social, exponiendo situaciones que podrían señalarse como dilemas. Un ejemplo podría ser cuando es descubierto el Templo Mayor mexica, cuando se realizaban las obras de excavación para el metro capitalino, a un costado del Palacio Nacional y la Catedral Metropolitana, propiciando el rescate de una parte del templo, a costa de inmuebles coloniales que fueron construidos sobre él, pero sin continuar a costa del Palacio o la Catedral. Entonces, en un mismo espacio, confluyeron tres planos históricos: prehispánico, colonial y contemporáneo.

\section{CONCLUSIONES}

Los centros históricos constituyen espacios y relaciones donde convergen procesos históricos que otorgan un valor identitario a aquellos elementos urbanos (edificaciones, monumentos y espacios abiertos) de mayor data. Pero adquiriendo nuevos sentidos y tensiones con los procesos que se van sucediendo, que van haciendo nuevas demandas para hacer funcional estos espacios en lo social, económico y político.

Su relevancia la adquieren en gran medida con base en las relaciones que establecen en la estructura urbana, a partir de su centralidad. Sin embargo, el tratamiento de la centralidad, predominantemente se da como un supuesto dado por ser el asentamiento original, sin referencia a planteamientos y modelos provenientes de los estudios espaciales, predominantemente cuantitativos y geométricos, pero que otorgan alguna racionalidad sobre las relaciones entre centro, centralidad y los otros puntos y áreas físicas. Pero, esa visión tiene como principales limitaciones que no incorpora la riqueza y complejidad de las relaciones sociales, económicas y políticas desde las subjetividades y estrategias de los actores. Por ello, es necesario recuperar planteamientos de los centros históricos desde los enfoques patrimoniales y monumentales, que de hecho son los predominantes.

El planteamiento del trabajo es que si asumimos la existencia no de un solo centro histórico sino la multiplicidad de ellos, se abren otras posibilidades interpretativas que le otorgan a estos espacios, relaciones en diferentes planos históricos, así como nuevas posibilidades de sentido para quienes habitan e intentan interpretar la historia desde los ojos del presente y, probablemente, pensando en un futuro que presentará nuevos retos que están llegando a un punto de inflexión de riesgos, tales como el ahondamiento de la desigualdad social, los problemas ambientales y el uso responsable de las tecnologías de la comunicación que, paradójicamente, podrían contribuir a una pérdida de significación de los espacios físicos y las relaciones cara-cara, por la virtualización del espacio y las personas. Entonces, la búsqueda de nuevos caminos para el abordaje de los centros históricos podría ser una tarea significativa para otorgar identidad y sentido a una población creciente individualizada y sin una clara idea del futuro. 


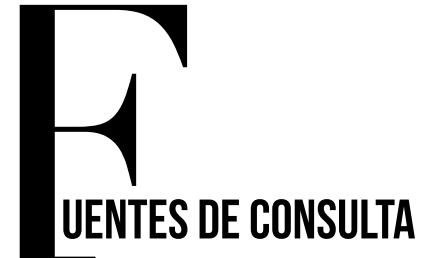

ATM (Arquitectos y Técnicos de Monumentos) (1964), Carta de Venecia 1964, Aprobada en el Segundo Congreso Internacional de Arquitectos y Técnicos de Monumentos, reunido en Venecia del 25 al 31 de mayo de 1964. Disponible en https://irp.mzcc2.org/upload/ secciones_archivos/o2-carta-devenecia1964_201901041854.pdf, consultado el 17 de abril de 2021.

Balandrano Campos, A., Valero Pié y Ziccardi, A. (coords.) (2016), Conservación y desarrollo sustentable de Centros Históricos, unAm, México. Disponible en http://www.red-centros-hist. unam.mx/assets/conservacion_y_desarrollo_sustentable_de_ centros_historicos.pdf, consultado el 17 de abril de 2021.

Bassols, Á. (1979), México formación de regiones económicas, UNAM, México.

Borja, J., Carrión, F., Corti, M. (eds.) (2016), Ciudades para cambiar la vida. Una respuesta a Hábitat III. Disponible en https://cafedelasciudades.com.ar/Ciudades_para_cambiar_ lavida_.pdf, consultado el 11 de abril de 2021.

Camagni, R. (2005), Economía urbana, Ed. Antoni Bosch, Universidad Autónoma de Barcelona, España.

Camallonga, J. (2012), Centros históricos: Análisis y perspectivas desde la geografía, Universidad de Alicante, España.

Carrión, F. (ed.) (2001), Centros históricos de América Latina y el Caribe, unESCo-BID-FLACso, Quito. Disponible en https://biblio.flacsoandes.edu.ec/shared/biblio_view. php?bibid=11257\&tab=opac, consultado el 15 de abril de 2021.

Carrión, F. (ed.) (2001), "Medio siglo en camino al tercer milenio: los centros históricos en América Latina", Centros históricos de América Latina y el Caribe, Quito: UNESCO-BID-FLACSO, pp. 29-93. Disponible en https://biblio.flacsoandes.edu.ec/shared/biblio_ view.php?bibid=11257\&tab=opac, consultado el 15 de abril de 2021.

Carrión, F. (2005), "El centro histórico como proyecto y objeto de deseo”, EURE, vol. XXXI, núm. 93, pp. 89-100.

Carrión, F. (2006), Lugares o flujos centrales: los centros históricos urbanos. Medio ambiente y desarrollo, CEPAL-Naciones Unidas Santiago de Chile, Chile.

Carrión, F. (2019), "El espacio público es una relación no un espacio". En Carrión Mena, F. y Dammert-Guardia, M. (eds.), Derecho a la ciudad. Una evocación de las transformaciones urbanas en América Latina, CLACSO, IFEA, Lima, pp. 191-219. Disponible en https://www.clacso.org. ar/libreria-latinoamericana/libro_detalle.php?orden=\&id libro=2055\&pageNum_rs_libros $=1 \&$ totalRows_rs_libros $=1431$, consultado el 30 de octubre de 2020.

Congreso Internacional de Arquitectura Moderna (CIAM) (1933), Carta de Atenas. Disponible en https://conservacion.inah. gob.mx/normativa/wp-content/uploads/Documento2991.pdf, consultado el 14 de abril de 2021.

Coulomb, R. (2001), "El Centro Histórico de la Ciudad de México: del rescate patrimonial al desarrollo integral”. En Carrión, F. (ed.), Centroshistóricos de América Latinay el Caribe, UNESCO-BID-FLACSO, Quito, pp. 139-155. Disponible en https://biblio.flacsoandes.edu. ec/shared/biblio_view.php?bibid=11257\&tab=opac, consultado el 15 de abril de 2021.
Coulomb, R. (2019), "Renovación urbana, políticas habitacionales y procesos de gentrificación en el centro histórico de la ciudad de México: mitos, conceptos y realidades". En Carrión Mena, F. y Dammert-Guardia, M. (eds.), Derecho a la ciudad. Una evocación de las transformaciones urbanas en América Latina, CLACSO, IFEA, Lima, pp. 113-136. Disponible en https://www.clacso. org.ar/libreria-latinoamericana/libro_detalle.php?orden=\&id_ libro=2055\&pageNum_rs_libros $=1 \&$ totalRows_rs_libros $=1431$, consultado el 30 de octubre de 2020.

Cuadrado-Roura, J. (2013), ¿Es tan "nueva” la "Nueva Geografia Económica"? Sus aportaciones, sus límites y su relación con las políticas, Universidad de Alcalá, Madrid, España, pp. 5-28.

De Mattos, C. A. (1999), "Teorías del crecimiento endógeno: lectura desde los territorios de la periferia", Estudios Avanzados, vol. 13, núm. 36, Universidad Católica de Chile, Chile. pp. 183-208.

Duch, Néstor B. (2008), La Teoría de la Localización. Universidad de Barcelona. Disponible en http://www.scribd. com/doc/4526619/Duch-Brown-La-teoria-de-la-localizacion, consultado el 9 de diciembre de 2020.

Gobierno de la Ciudad de México (2017), Constitución Política de la Ciudad de México, Gaceta Oficial de la Ciudad de México, 5 de febrero de 2017 (Última reforma: 27 de noviembre de 2019). Disponible en https://www.scjn.gob.mx/sites/default/files/ justicia_constitucional_local/documento/2020-01/118922.pdf, consultado el 17 de abril de 2021.

González-López, S. (2004), "La reestructuración económicoterritorial de la megalópolis de México ante la globalización", Revista Urbano, vol. 7, núm. 10, Universidad del Bío Bío Concepción, Chile. pp.75-84.

Krafta, R. (2008), "Fundamentos del análisis de centralidad espacial urbana”, Revista Centro, Organización Latinoamericana y del Caribe de Centros Históricos-OLACCHI, núm. 2, pp. 15-28.

Krugman, P. (200o), "Where in the world in the New Economic Geography", The Oxford Handbook of Economic Geography, Oxford University Press, Oxford.

Lezama, J. (2010), Teoría social, espacio y ciudad, El Colegio de México, México.

Mediateca INAH (1929), Plano de la Ciudad de México. Disponible en https://mediateca.inah.gob.mx/islandora_74/islandora/ object/mapa\%3A151, consultado el 11 de abril de 2021.

Organización de las Naciones Unidas (ONU) (2016), Nueva Agenda Urbana, A/RES/71/256. Disponible en https://onuhabitat.org. $\mathrm{mx} /$ index.php/la-nueva-agenda-urbana-en-espanol, consultado el 9 de diciembre de 2019

Pineda, J. (2010), Desarrollo y espacio regional, una aproximación teórico-metodológica, Ed. Espacios públicos, unAM, México.

Rodríguez, P. (2008), El centro histórico: del concepto a la acción integral, Organización Latinoamericana y del Caribe de Centros Históricos-OLACCHI Quito, Organismo Internacional.

Soja, E. (2008), Postmetrópolis. Estudios críticos sobre las ciudades y las regiones, Ed. Traficantes de sueño, Madrid.

Soltero, G. (2009), "Identidad narrativa y el centro histórico (de la Ciudad) de México", Andamios. Revista de Investigación Social, vol. 6, núm. 12, pp. 133-153. Universidad Autónoma de la Ciudad de México, Distrito Federal, México. Disponible en http://www.redalyc. org/articulo.oa?id=62815957007, consultado el 11 de abril de 2021.

UNESCO-PNUD (1977), Conclusiones del Coloquio sobre la preservación de los Centros Históricos ante el crecimiento de las ciudades contemporáneas, Quito, Ecuador, 11 de marzo de 1977. Disponible en https://ipce.culturaydeporte.gob.es/eu/dam/ jcr:da21dfac-4e15-4937-bd6a-d6ead67155be/1967-carta-dequito.pdf, consultado el 17 de abril de 2021. 